\title{
An Acceptance Attitude Model and Empirical Analysis Towards Online Tourism Service
}

\author{
Hui Zheng ${ }^{a}$, Fei Long ${ }^{b, *}$, Wanhua Yang ${ }^{a}$, Xiangrong Cheng ${ }^{c}$ \\ ${ }^{\text {a }}$ School of Tourism Management, Hunan University of Commerce, China \\ ${ }^{\mathrm{b}}$ Department of Economics and Management, Changsha University, China \\ ${ }^{\mathrm{c}}$ College of International Studies, Hunan University of Commerce, China \\ *Corresponding author: Fei Long, Doctor, lf831028@sohu.com
}

\begin{abstract}
With the increasing development of Internet, more and more customers know and order travel services and its supporting services through the network; however the certain attitude formed by customers during understanding and accepting online tourism services, largely determines subsequent user behavior, and leads the development direction of online tourism services. Then, an acceptance attitude model of online tourism services and other assumptions are presented based on the attitude data of online tourism services for different customers, and subsequently the empirical analysis is conducted by using mathematical tools and analysis. The empirical results show that: improving interactive channels and efficiency of online tourism services is the core of online tourism service innovation, which will lead the direction of online tourism service innovations.
\end{abstract}

Keywords: online tourism service; information technology adoption; acceptance attitude model; validity check; service interaction

\section{Introduction}

The attitude, as a person's inner state, is the tendency of things a person likes and dislikes, and it tends to be the pilot of individual behavior, also is an important indicator to predict individual behavior [1, 2]. With the increasing development of Internet, more and more people come to know through the internet and order tourism and support services, therefore, the attitudes formed in understanding and accepting online tourism services by customers influence the customers' subsequent use behavior at a large extent, and determine the development direction of online tourism services [3]. To maintain and change the behavior tendencies of customers, we must maintain and change the customers' attitude, which will be a foundation and direction for tourism service innovation! 
Presently, the studies on recommendation acceptance at home and abroad are mostly based on the technology acceptance theory. It can be said that the acceptance and adoption towards information technology has been the focus of attention, and its history has been over twenty years abroad. Davis proposed the technology acceptance model(TAM) in 1989, which summarized the factors that influence individual IT as perceived ease of use, perceived usefulness, attitudes, intentions and actual behavior, and built a logic structure for describing the relationship of these factors [4]. Perceived usefulness means that users believe that the use of the system will increase job performance, and perceived usefulness and perceived ease of use is also affected by external variables [5], which include system characteristics, user characteristics, task characteristics, organizational characteristics, management characteristic, and so on [6].

Sally Rao and Troshani studied the motivation users use the online service, and they proposed the user behavioral models of online services based on the scalable TAM [7]. Lin Jiabao and etc. conducted the study on perceived ease of use, perceived usefulness and safety, information quality, convenience and trust tendency, and did some research on user usage behavior [8]. Ling Hong, Xia Li and etc. studied the mobile business user adoption of content delivering category, and validated the proposed models and assumptions [9]. The results show that the TAM is suitable to explain the mobile commerce user adoption of content delivery class.

In this paper, we conduct a detailed study for the attitudes of online tourism service at all levels within the specified region by collecting the evaluation attitudes towards online tourism service of 298 customers in different categories.

\section{Building an Attitude Model}

The existing research on IT acceptance and adoption provides a basic perspective for this paper. However, as for the online tourism recommendation service that is an online decision support system, it is insufficient especially for the mechanism factors of consumers own, it is still necessary to strengthen customer acceptance attitudes from the legitimacy, effectiveness and fairness of online tourism services.

Legitimacy: The legitimacy concerns the legitimacy of the political system that is how to ensure that the rights of individual freedom, we propose the following assumptions:

$\mathrm{H} 1$ : The online tourism services with the legal validity will have a significant positive impact on the customer acceptance attitude

(2) Effectiveness: Effectiveness is the most important criteria for judging whether the public action of service management organization is successful, that is what we call social benefits. Effectiveness shows that a public action achieves the purpose, although the cost will also become an important criterion to consider, but the effectiveness can often be independent of the cost. If a public action does not meet the intended purpose, even if the paid cost is rarely, this public action will mean nonsense [9]. Based on this, we propose the following assumptions: 
$\mathrm{H} 2$ : The online tourism services with the effectiveness will have a significant positive impact on the customer acceptance attitude

(3) Irritation: If people feel the online tourism service reduces the efficiency, and even hurt their dignity, which will have a greater negative impact on the acceptance attitude of customers. If the tourism service makes things even more troublesome and unpleasant, the customer may be reluctant to accept the online tourism services. In addition, the customers with different levels of knowledge will have different acceptance attitudes toward online tourism service [10]. Based on this, we propose the following assumptions:

H3: The online tourism services with i Irritation will have a negative impact on the customer acceptance attitude

(4) Credibility: Credibility means that customer perception for online tourism services is real and credible. The credibility of online tourism services will be affected by many factors, especially the business credibility and information transfer. At the same time, it also is affected by online tourism service media, for example, from the study on online tourism services in the past, we found that the credibility of is lower than that of publications of travel services unless online tourism services spread by a good brand. Based on this, we propose the following assumptions:

H4: The online tourism services with credibility will have a negative impact on the customer acceptance attitude

(5) Fairness: The fairness contains three meanings: the first means that personal contribution and benefits is equal, which is also known as the fiscal balance. We propose the following assumptions:

H5: The online tourism services with fairness will have a negative impact on the customer acceptance attitude

(6) Interactivity: From the interactive purpose between travel service providers and customers, the interactive platform is interactive and communicative channels for enhancing customer satisfaction with service management organization. Online interactivity is an effective way of "face to face" communication to serve the public and regulatory agencies. Based on this, we propose the following assumptions:

H6: the online tourism services with interactivity will have a negative impact on the customer acceptance attitude

From the above analysis and assumptions, this paper proposes an acceptance attitude model of online tourism service, as showed in Fig.1.

In this paper, the analysis data comes from the questionnaire survey, and the questionnaire consists of two parts: the first part is the target demographic survey variables, including age, sex, occupation, education level, favorite online tourism services and the use time and frequency of online tourism services; the second part covers all the variables included in the measure items of empirical model. 


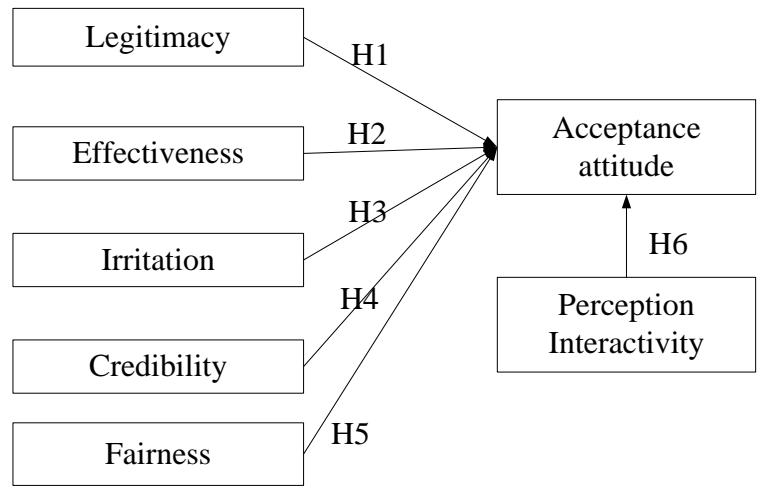

Fig.1 - The acceptance attitude model towards online tourism service

\section{Questionnaire Design and Collection}

\subsection{Questionnaire design}

This data comes from the survey, and the empirical methods are used to validate the proposed model. In this paper, questionnaire design consists of two parts, the first part includes the target demographic survey variables, including age, sex, occupation, education level, favorite online tourism services and the use time and frequency of online tourism services; the second part covers all the variables included in the measure items of empirical model. In this survey, we distributed 478 questionnaires and 347 copies were returned. We collect 298 valid questionnaires expect incomplete and failed ones, which account for $62 \%$ of the total sample.

\subsection{Sample collection}

The questionnaire distribution methods convers the direct filling, QQ transferring, e-mail and etc. and the people filling the questionnaires include the students of tourism school, related professional students and other levels social personnel, and all of them have the online tourism service experience. The sample statistics these characteristics of the recycling sample, and we can see that most of the sample is below 35 years old, men account for $59.40 \%$ and women account for $40.60 \%$. Most of them are subject to a college education. In this survey, enterprise employees account for $33.9 \%$, students account for $25.5 \%$, and the objects with a monthly income more than 1500 account for 53\%, the form of online tourism services mostly used is tourism service inquiry, followed by online service of personal/enterprise, the use of complaints or report is $6 \%$ and the use of economics/tourism/cultural information accounts for $3 \%$.

Descriptive statistics describe the variables by the mean value, arithmetic sum, standard deviation, maximum, minimum, variance, standard error of the range and average value, Through the $\mathrm{Z}$ fraction, that is

$$
\mathrm{Z}_{i}=\frac{x_{i}-\bar{x}}{s}
$$

Where $\mathrm{xi}$ is the $\mathrm{i}$-th observation value of the variable $\mathrm{x}, \bar{x}$ is an average value of the variable $\mathrm{x}, \mathrm{S}$ is the standard deviation of the variable $\mathrm{x}$. 


\section{Data analysis}

In this part, the two methods of exploratory factor analysis and confirmatory factor analysis are used to examine the reliability and validity of the measurement model, and finally the proposed model and assumptions are verified using structural equation model with SPSS13.0 and PLS-Graph.

\subsection{Reliability check}

Thee liability means the measurement credibility degree, and shows mainly the consistency and stability of measurement results. Therefore, a good measuring tool should always keep stable for repeated measurement results, which is proved to be credible. The reliability is the ratio of true variance of a set of measurement points to the total variation, that is

$$
r_{x x}=\frac{s_{r}^{2}}{s_{x}^{2}}
$$

$r_{x x}$ denotes the reliability factor, sr denotes the true variance, sx denotes the total variance. The $\alpha$ reliability coefficient is the most commonly used reliability coefficient, which indicates the score consistency among item scores for each question, and is suitable for the questionnaire survey, can be used to accumulate the reliability of Likert-type scale. The cronbach formula of $\alpha$ may be described below:

$$
\alpha=\frac{k}{k-1}\left(1-\frac{\sum_{i=1}^{k} s_{i}^{2}}{s_{x}^{2}}\right)
$$

In the above equation, $\mathrm{k}$ is the question number of quiz, si is the score variance of $\mathrm{i}$-th question, sx is the variance of test scores. When the reliability coefficient of this test results $\alpha>$ 0.7 , we may determine the investigation materials will be reliable and we can make the next step.

\subsection{Validity check}

Factor analysis is the analytical methods to find some dominate underlying factors that cannot be directly observed among the variables with the associate relationship. The factor analysis can be used in the following two aspects: the one is to seek the basic structure and find less meaningful factor to reflect the basic structure of the data by factor analysis. The other is to represent the original data structure with less structure invariable, and keep the original data structure information. In this paper, the principal component analysis is used to conduct factor analysis, and the factors of characteristic values greater than 0.56 are selected, the common factor is extracted by using the maximum variance rotation. 


\section{Analysis and Conclusion}

Based on the recycling questionnaire data, we use SPSS13.0 to conduct principal component analysis for the data after reliability examination optimization, and the KMO value of sample is 0.958 after the principal component analysis through maximum variance orthogonal rotation. Because the value of KMO is closer to 1, the correlation among the item variables is better and is more suitable for factor analysis. Bartlett spherical examination determines whether the correlation matrix is a unit matrix, indicating whether the factor model is outdated. Finally, we obtain 7 factors with the eigenvalues greater than 0.56 by using SPSS principal component extraction and variance maximum rotation, which indicates that the factor structure is very clear.

After testing the structural model with PLS-Graph3.0, the hypothesis examination results are showed in Fig. 2:

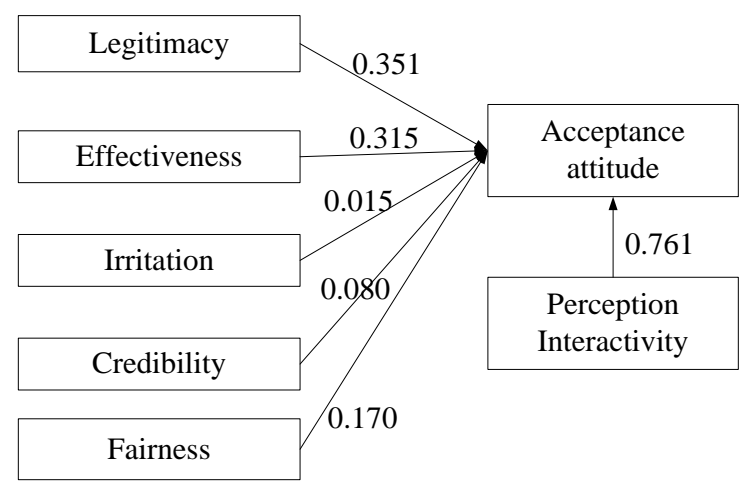

Fig. 2 - The hypothesis examination results

The analysis shows that in our proposed six assumptions, there are four ones being significant under the level of $\mathrm{P}$ valued as 0.05 , and another two are not significant at the level of $\mathrm{P}$ valued as 0.05 . That is to say the legitimacy, effectiveness and fairness may exert a significant effect on the user attitudes, and the perceived interactivity is also significant to affect the user attitude, while the impact of irritation and credibility on user attitudes is not significant.

(1) Interactivity $(\beta=0.761)$ is the most important factors that affecting the online tourism service attitude of customers, which indicates that the customers is very serious about the interactivity of online tourism service. Therefore, a primary aspect of the travel service innovation is to optimize the interactive channel between online tourism services provider and customers, and improve the interactive efficiency between tourism service providers and customer.

(2) Legality $(\beta=0.351)$ is the second most important factor affecting the acceptance attitude towards online tourism service. This shows that the legality will exert a important impact on acceptance attitude towards online tourism service.

(3) The effectiveness $(\beta=0.315)$ is the third most important factor affecting the acceptance attitude towards online tourism service and this effectiveness will strengthen the positive attitude to online tourism services. 
(4) The fairness $(\beta=0.170)$ exerts a positive influence on user acceptance attitudes towards online tourism services. Online tourism service provider should improve the service fairness, which will have a positive effect on the acceptance attitude towards online tourism service.

(5) The irritation ( $\beta=-0.015$ ) is not significant to affect user acceptance attitude towards online tourism services, and it exert a negative impact on online tourism service attitude, which is consistent with the hypothesis of this paper. Once the online tourism service cannot provide the user the desired functionality, so that customers are tired to this, which will enable customers to produce anxiety, and have a negative attitude on acceptance attitudes.

(6) The credibility $(\beta=0.080)$ is not significant to affect user acceptance attitude towards online tourism services. The legality of the investigation in this study has covered part of the trusted content, so that everyone believe that content of online tourism services is credible in the sample survey.

\section{Acknowledgement}

The authors would like to thank the referee. This work is partly supported by NFC of China under Grant No.61304184, by Hunan Provincial Natural Science Foundation of China (2016JJ6010) and Social Science Project of Hunan provincial education office (11C0752).

\section{References}

1. Y.Y. Zhang, Q.J. Hu, J. Gu, Study on Family Tourists' Perception and Behavior Attitude in the International Tourism Festival. Soft Science, 2012, pp. 135-140.

2. K. Bai, Y.F. Ma, T.S. Li, T.Y. Sun, An Association Study on Tourists' Cognition, Perceived Value and Behavior Intention Based on Structural Equation Mode l (SEM). Journal of Geography, 2010, pp. 244-253.

3. L. H. Major and R. Coleman, Source credibility and evidence format: Examining the effectiveness of HIV/AIDS messages for young African Americans. Journal of Health Communication: International Perspectives, 2012, pp. 515-531.

4. F.D. Davis, Perceived Usefulness, Perceived Ease of Use, and User Acceptance of information Technology. MIS Quarterly, 1989, pp. 319-339.

5. H. Rong, S. Huo, et al. User similarity-based collaborative filtering recommendation algorithm. Journal of Communications, 2014, pp. 16-24.

6. X. Peng, Z. Feng, X. Sun, Q. Zhu, The Empirical Research on Users' Continuance Intention in Microblogging Services. New Technology of Library and Information Service, 2012, pp.78-85.

7. S. Rao, I. Troshani, A Conceptual Framework and Propositions for the Acceptance of Mobile Services. Journal of theoretical and applied electronic commerce research, 2007, pp. 61-73.

8. J.B.Lin, Y.B. Lu, J.L. Zhang (2009). An Empirical study on Consumers' Trust of Mobile Stock Based on TAM Theory. Journal of Management Science, 2009, 22(5): 61-71.

9. L. Hong, X. Li, F.H. Zeng, Research on the Acceptance Model of Content Delivery 
Mode Mobile Commerce. Journal of Management Science of Shanghai, 2008, pp. 31-35.

10. R.K. Chellappa, and R.G.Sin, Personalization versus Privacy:An Emprical Examination of the Online Consumer's Dilemma. Information Technology and Management, 2005, pp.181-202. 